\title{
Entwicklung und Praxis der Kognitiven Psychotherapie
}

\author{
Schweizerischer Verein für Kognitive Psychotherapie (SVKoP)
}

A. Souche, R. Zumbrunnen

La version française a paru

dans le $n^{\circ} 14 / 2002$
Korrespondenz:

Dr. med. Alain Souche

Route de St-Julien 176

CH-1228 Plan-les-Ouates

E-Mail: asouche@iprolink.ch

\section{Grundzüge der Kognitiven Therapie}

Die Kognitive Therapie, auch Kognitive Verhaltenstherapie (KVT) genannt, verbindet die vom Behaviorismus in den 50er und 60er Jahren entwickelten verhaltenstherapeutischen Ansätze mit kognitiven Behandlungstechniken. Die Bedeutung gedanklicher Prozesse wurde erstmals von den amerikanischen Klinikern A. T. Beck und A. Ellis im Zuge der sogenannten kognitiven Wende in den 70er Jahren hervorgehoben. Innerhalb der Kognitiven Therapie lassen sich heute mehrere Arbeitsansätze unterscheiden. So konzentrieren sich einige Behandlungsmodelle auf verhaltensbezogene Techniken wie beispielsweise auf die Konfrontation mit angsterregenden Situationen, die Bewältigung konkreter Aufgaben oder die Lösung aktueller Probleme, während andere Therapeuten den Einsatz spezifisch kognitiver Techniken wie zum Beispiel die Identifizierung und Veränderung selbstschädigender Gedanken und Denkmuster in den Vordergrund stellen. Jüngere, namentlich in Italien entwickelte Ansätze wiederum betonen bislang weniger berücksichtigte Aspekte wie beispielsweise die Beziehung zwischen dem Patienten und dem Therapeuten, die persönliche Lebensgeschichte des Patienten sowie die in der Gesprächssitzung erlebten Emotionen.

Die KVT als Therapieverfahren begreift das Gespräch zwischen dem Patienten und dem Therapeuten als Mittel zur Evaluation und Behandlung psychischer Störungen. Sie beschränkt sich dabei jedoch nicht auf eine blosse «Diskussion», sondern fordert den Patienten zur aktiven Mitarbeit an konkreten Aufgabenstellungen auf. So beispielsweise wird der Patient angehalten, seine Selbstbeobachtungen in einem Tagebuch aufzuzeichnen, aktiv an der Veränderung irrationaler Einstellungen zu arbeiten, sich stärker in für ihn problematischen Bereichen zu engagieren oder sich gefürchteten Situationen bewusst zu stellen. Der Psychotherapeut seinerseits beschränkt sich nicht darauf, dem Patienten zuzuhören. Vielmehr greift er in den Behandlungsprozess ein, um den Patienten zu befähigen, sich auf ein oder mehrere Schlüsselprobleme zu konzentrieren und schrittweise Alternativen $\mathrm{zu}$ seinem unangemessenen Verhalten und Denken zu entwickeln.

Die KVT geht davon aus, dass psychische Probleme zumindest in gewissem Grade in angeeignetem, das heisst erlerntem Verhalten begründet sind. Der Begriff Verhalten wird hier breit gefasst und schliesst neben den physiologisch-körperlichen Aktivitäten auch kognitive und emotionale Aspekte ein. Diesem Ansatz folgend muss der Therapeut die Lernprozesse kennen, welche die Entstehung, die Aufrechterhaltung und das Verlernen von Verhalten steuern, und er muss in der Lage sein, diese in der Therapie zu nutzen. In diesem Zusammenhang wird zwischen reaktivem (pavlovischem), operativem (skinnerschem), sozialem (nachahmendem) und kognitivem Lernen unterschieden. In der Therapie erlaubt die Ortung dieser komplexen Prozesse die Bildung von Hypothesen über die Entstehung und Aufrechterhaltung psychischer Probleme. Ferner liefern diese Prozesse einen wertvollen Schlüssel für die Durchführung der Behandlung.

Die kognitiven Aspekte des Verhaltens spielen bei der Entstehung und Aufrechterhaltung psychischer Störungen eine zentrale Rolle. Zur Kognition gehören unsere Gedanken, Vorstellungen und Interpretationen über alles, was uns umgibt und was in uns vorgeht. Die KVT greift das Gedankengut des antiken Philosophen Epiktet auf, der postulierte, die Menschen seien weniger durch die Dinge selbst verwirrt, beunruhigt und erschüttert, als durch die Art, wie sie über diese denken. Stellen Sie sich einen Menschen vor, der sich abends alleine zu Hause befindet und ein lautes Geräusch hört. Denkt er nun, Diebe seien in seine Wohnung eingebrochen (Kognition), so wird er unruhig und hat Angst (Emotion) und ruft vielleicht die Polizei (Aktion). Vermutet er hingegen, er habe aus Unachtsamkeit das Fenster offengelassen (Kognition), so bleibt sein Gefühlszustand neutral (Emotion) und er schliesst einfach das Fenster (Aktion). Die gleiche Sequenz gilt für das Auftreten psychischer Störungen und, bis zu einem 
gewissen Grade, auch für die Entstehung physischer Erkrankungen. Irrige Annahmen oder unangemessene Denkmuster führen zu einer pathologischen und selbstschädigenden Verarbeitung von Informationen, die ihrerseits zur Entstehung oder Aufrechterhaltung eines klinischen Syndroms beiträgt. Als Beispiel könnte hier die falsche Interpretation natürlicher Vorgänge wie beispielsweise des Herzklopfens angeführt werden, das bei gewissen Personen eine Panikattacke auslösen kann.

Während des gesamten Behandlungsprozesses werden der Identifizierung und der detaillierten Analyse der drei zentralen Aspekte der menschlichen Erfahrung, namentlich die Gedanken, die Gefühle und die Handlungen, besondere Aufmerksamkeit geschenkt. Diese drei Erfahrungsebenen sind eng miteinander verknüpft, und das Therapieergebnis hängt von der Fähigkeit des Patienten ab, in den drei Bereichen alternative Lösungen zu entwickeln und diese an die Stelle der gewohnten Reaktionen zu setzen.

Die KVT zeichnet sich durch das kontinuierliche Bestreben aus, Hypothesen und Therapieergebnisse systematisch zu evaluieren. Unter den zahlreichen praktizierten psychotherapeutischen Ansätzen stellt die KVT jene Methode dar, deren therapeutische Wirksamkeit heute am weitesten wissenschaftlich abgesichert ist. Das Anliegen, die eigene Effizienz zu überprüfen, findet sich auch im Therapieprozess selbst wieder: In einem vom Patienten und Therapeuten gemeinsam erarbeiteten Therapievertrag werden konkrete Ziele definiert, die als Kriterien für die Auswertung der Behandlung dienen.

\section{Indikationen der Kognitiven Verhaltenstherapie}

Die Indikationen der KVT hängen neben der Diagnose auch von anderen Kriterien ab. Aus diagnostischer Sicht ist die Wirksamkeit der Kognitiven Therapie in erster Linie bei der Behandlung von Angststörungen wissenschaftlich nachgewiesen. So wurden Erfolge bei der Behandlung von spezifischen Phobien (Höhe, Tiere, Wasser, usw.), von Panikreaktionen einschliesslich Agoraphobie sowie von sozialen Phobien erzielt. Auch bei in der Regel nur schwer behandelbaren Zwangsstörungen ist eine Kognitive Verhaltenstherapie indiziert. Bislang liegen nur geringe Erfahrungen bei der Behandlung generalisierter Angststörung und Hypochondrie vor.

Mittelschwere Depressionen können ebenfalls in einer KVT behandelt werden. Bei schweren Depressionen hingegen müssen medikamen- töse Kombinationstherapien Anwendung finden, während leichte Depressionen in der Regel keiner spezialisierten Behandlung bedürfen.

Die Wirksamkeit der Kognitiven Verhaltenstherapie bei der Behandlung weiterer Störungsbilder wird derzeit untersucht, und die Ergebnisse sind vielversprechend. So beispielsweise haben Kontrolluntersuchungen Therapieerfolge im Falle von Bulimie, pathologischer Spielsucht und suizidalen Tendenzen bei Borderline-Patienten bestätigt. Ferner sind die Modelle zur Behandlung von Suchterkrankungen (Alkoholismus, Toxikomanie), Schizophrenie sowie von sexuellen und psychosomatischen Störungen häufig von den Methoden der Kognitiven Verhaltenstherapie inspiriert. Auch bei der Behandlung von Kindern und älteren Menschen findet die KVT in zunehmendem Masse Anwendung.

Nicht nur das Störungsbild, sondern auch die Therapiemotivation entscheidet, ob eine KVT indiziert ist. Zur Erreichung optimaler Behandlungsresultate muss der Patient bereit sein,

- sein Verhalten und seine Denkweisen wirklich ändern und nicht nur die Ursachen seiner Probleme verstehen zu wollen;

- aktiv mit dem Therapeuten zusammenzuarbeiten;

- sich auf ein oder wenige Schlüsselprobleme zu beschränken, da die Therapie einen begrenzten Zielkatalog verfolgt und zeitlich limitiert ist.

\section{Therapeutische Methoden}

Eine Kognitive Verhaltenstherapie besteht in der Regel aus 10 bis 30 Gesprächssitzungen, die sich über einen Zeitraum von 4 bis 12 Monaten erstrecken. Der Therapeut und der Patient identifizieren gemeinsam die zu behandelnden Probleme und legen die Therapieziele fest. Der Patient trainiert die im Rahmen einer Gesprächssitzung absolvierten praktischen Übungen entweder zu Hause oder in der problematischen Situation selbst. So beispielsweise ist ein an Agoraphobie leidender Patient aufgerufen, seine Angst durch einen Gang ins Kaufhaus zu konfrontieren. Das selbständige Training zu Hause ist ein bedeutender Bestandteil der Therapie, denn nur auf diese Weise kann das in der Gesprächssitzung erlernte alternative Verhalten konsolidiert werden. Die Rolle des Therapeuten im Behandlungsprozess ist die eines Wegweisers und Trainers. Er kann dem Patienten Erläuterungen anbieten, die diesem helfen, das Bewusstsein über seine Störung zu schärfen. Hierbei muss der Therapeut lehrerhaftes, autoritäres Auftreten 
vermeiden und es dem Patienten überlassen, mit Hilfe der vom Therapeuten geleiteten «sokratischen» Befragung neue Wege zu erschliessen, die er beschreiten kann und will. Die Behandlung kann ferner durch die Lektüre von Self-Help-Leitfäden über die spezifische Störung des Patienten ergänzt werden.

\section{Die KVT im Kreuzfeuer}

Die Kognitive Therapie wird von verschiedensten Seiten, in erster Linie aber von den Vertretern anderer psychotherapeutischer Ansätze, kritisiert. Die am häufigsten angeführten Argumente, deren Vertreter leider meist nur eingeschränkte Kenntnisse über den wahren Charakter der Kognitiven Therapie besitzen, sind folgende:

Die Kognitive Verhaltenstherapie

- simplifiziert komplexe Zusammenhänge, andere Kritiker wiederum werfen der KVT vor, zu kompliziert zu sein;

- verbleibt auf der Ebene der Symptome, ohne die Wurzeln der Störungsbilder in Angriff zu nehmen;

- verschiebt Probleme, ohne sie wirklich zu lösen;

- «normalisiert» den Patienten gemäss den Bedürfnissen der Gesellschaft;

- wendet standardisierte Rezepte an;

- gibt vor, alle Probleme lösen zu können, andere Kritiker hingegen halten die KVT für eine Therapie der zweiten Wahl, für eine Notlösung für jene Patienten, denen die Gabe der Selbstbeobachtung fehlt;

- schenkt emotionalen Aspekten zu geringe Beachtung;

- lässt die subjektiven Faktoren des Therapeuten unberücksichtigt.

Eine Betrachtung der Tatsachen aber lässt rasch erkennen, dass diese stereotype Kritik von der von Therapeuten und Patienten täglich erfahrenen Therapierealität meilenweit entfernt ist. Der eigentlichen Therapie geht stets eine sorgfältige, in Anamnese, Diagnose und funktionale Analyse der Störung gegliederte Evaluation voraus. Diese einzelnen Schritte werden offen dargelegt und mit dem Patienten gemeinsam besprochen. Auf diese Weise wirkt der Patient in voller Kenntnis der Lage aktiv bei der Ausarbeitung des Therapieplans mit. Die Therapie nimmt nicht die «oberflächlichen» Symptome in Angriff, sondern ist auf die Veränderung jener Probleme gerichtet, die den Patienten veranlassen, Hilfe zu suchen.
Allein schon die Gegenüberstellung von «eigentlicher Ursache» und «oberflächlichen» Symptomen ist problematisch, denn die Medizin wird sich in zunehmendem Masse bewusst, dass Kausalzusammenhänge meist kreisförmig, nicht linear verlaufen. So sind beispielsweise die aktuellen Einstellungen und Verhaltensweisen des Patienten sowohl Ursache als auch Folge der Störung, tragen sie doch dazu bei, diese auszulösen und aufrechtzuerhalten. Auch zollt die KVT dem Patienten insofern hohen Respekt, als sie direkt auf die vom Patienten erwünschte Überwindung seines aktuellen Leids gerichtet ist. Sie ist kein Standardmenü, sondern ein an den persönlichen Bedürfnissen des Patienten ausgerichtetes Behandlungsmodell, das periodisch evaluiert und bei Bedarf angepasst werden kann. Der Therapeut spielt mit offenen Karten und tauscht sich mit dem Patienten über die Hypothesen und Therapieziele aus. Wirksamkeit und Zweckmässigkeit der KVT hängen nicht von dem kulturellen Hintergrund des Patienten ab, sondern entwickeln sich entsprechend der Motivation und Fähigkeit des Patienten, sich seinen Schwierigkeiten zu stellen. Die KVT ist keine universelle Antwort auf alle Krankheitsbilder, sie ist vielmehr eine Art empirische Partnerschaft, welche die bereits erreichten Erfolge und die noch zu bewältigenden Aufgaben kontinuierlich evaluiert. Im Gegensatz zu den meisten anderen psychotherapeutischen Methoden gelingt es der KVT seit mehreren Jahren, ihre Wirksamkeit bei der Behandlung einer zunehmenden Anzahl von Krankheitsbildern wissenschaftlich $\mathrm{zu}$ untermauern.

Jede Psychotherapie muss die Gefühle des Patienten und des Therapeuten im Behandlungsprozess berücksichtigen. Der durch Guidano und Liotti vertretene konstruktivistische Ansatz innerhalb der kognitiv-behavioralen Schule wirft der klassischen Kognitiven Therapie eine Überbetonung der Rationalität vor und stellt ein Modell vor, welches die emotionalen Aspekte stärker in die Behandlung integriert. Hier werden die Gefühle nicht als blosse Nebenerscheinung grundlegenden Denkens verstanden, sondern als eigenständige, verändernde Kraft im Netz der Ereignisse.

Dem Argument, die KVT liesse die subjektiven Faktoren des Therapeuten unberücksichtigt, soll entgegengehalten werden, dass im Rahmen der Therapieausbildung irrationale Denkweisen und Beziehungsstile des Therapeuten durchaus problematisiert und mit Hilfe von Supervisionen und Intervisionen aufgearbeitet werden. 


\section{Der Schweizerische Verein für Kognitive Psychotherapie (SVKoP)}

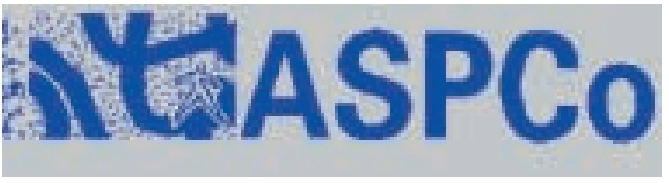

Dem auf Initiative von Lucio Bizzini 1994 in Yverdon-les-Bains gegründeten Verein gehören Psychiater und Psychologen an. Der SVKoP bezweckt,

- die Reflexion über die klinischen und theoretischen Aspekte des kognitiven Therapieansatzes zu fördern;

- Begegnungen zwischen Personen zu fördern, die einer beruflichen Tätigkeit im Bereich der Kognitiven Therapie nachgehen;

- Grund- und Weiterbildungsmöglichkeiten anzubieten;

- auf nationaler und internationaler Ebene Kontakte zu knüpfen und wissenschaftliche Symposien zu organisieren;

- Forschungstätigkeiten im Bereich der Kognitiven Therapie zu fördern.

Heute ist der SVKoP Ort des Austauschs und Bildungträger für Praktiker. Über diese Aktivitäten hinaus informiert der Verein Institutionen und die Öffentlichkeit über therapiebezogene Themen. Allerdings ist seine Tätigkeit nach wie vor zu stark auf die Westschweiz beschränkt, und die Zahl der Deutsch und Italienisch sprechenden Mitglieder ist leider immer noch sehr gering.

\section{Die Aktivitäten des SVKoP}

Im Laufe seines achtjährigen Bestehens hat der Verein zahlreiche regionale und internationale Kongresse organisiert beziehungsweise an ihrer Organisation teilgenommen. Mehrere Vereinsmitglieder haben Werke über den kognitiven Therapieansatz veröffentlicht. ASPCo Info, Informationsbulletin und Plattform für den professionellen Austausch, wurde bislang neunmal aufgelegt. ASPCo Info erscheint dreimal pro Jahr und enthält neben allgemeinen Informationen auch Beiträge zu spezifischen Themen. Eine Ausgabe behandelt eine ausgewählte Fragestellung, eine Nummer stellt die am jährlichen Kliniktag von in der Ausbildung befindlichen Mitgliedern präsentierten Arbeiten vor, und die letzte Ausgabe des Jahres konzentriert sich in erster Linie auf die Arbeiten der Vereinsmitglieder.

Das Angebot von Ausbildungsmöglichkeiten im Bereich der Kognitiven Therapie zählt zu den statutarisch festgelegten Aufgaben des Vereins. Die Ausbildung umfasst zwei Abschnitte: die Grundausbildung und die Fortbildung (Weiterbildung).

\section{Grundausbildung}

Die Grundausbildung wurde im Jahre 1996 aufgenommen. Zunächst erstreckte sie sich über einen Zeitraum von zwei Jahren, 2000 wurde dieser Zeitraum auf drei Jahre ausgedehnt. Im Zuge dieser Verlängerung wurde die Rolle der praktischen und klinischen Ausbildung erheblich aufgewertet. Das erste Jahr der Grundausbildung konzentriert sich auf die theoretischen und klinischen Grundlagen der Kognitiven Therapie. Im zweiten und im dritten Jahr dann stehen spezifische Krankheitsbilder und Zielgruppen einschliesslich der Gruppe der «schwierigen» Patienten im Mittelpunkt. Insgesamt umfasst die dreijährige Grundausbildung 300 Stunden. Ferner sieht die Ausbildung des SVKoP auch die Arbeit des Therapeuten an sich selbst sowie die Teilnahme an Supervisionen vor.

\section{Fortbildung}

Die Fortbildung findet in praktisch orientierten Fortbildungsseminaren statt, die jeweils einen bis drei Halbtage dauern. Im Zeitraum 2001-2002 wurden insgesamt 110 Stunden Fortbildung angeboten. Das Ausbildungsangebot des SVKoP wird von den Berufsverbänden der Schweizer Psychiater (SSPP) und Psychologen (FSP) anerkannt. Die Fortbildungsseminare stellen ein Forum für den Austausch professioneller Erfahrungen und Reflexionen dar. Sie bieten den Teilnehmern die Gelegenheit, sich über die unterschiedlichen Ansätze innerhalb der kognitiv-behavioralen Therapie zu verständigen und eigene Kenntnisse und Fähigkeiten in den Dienst der Kollegen zu stellen.

Die Ausbildungskurse des SVKoP werden von lokalen medizinischen und psychologischen Fachkräften, von in der KVT erfahrenen Therapeuten sowie von international angesehenen ausländischen KVT-Experten durchgeführt.

\section{Schlussfolgerungen}

Die vor rund dreissig Jahren entwickelte Kognitive Therapie, auch Kognitive Verhaltenstherapie (KVT) genannt, ist ein Behandlungsmodell, dessen Wirksamkeit bei der Behandlung von Angststörungen wissenschaftlich untermauert ist. Auch Depressionen und andere psychische Störungen können mit der KVT erfolgreich behandelt werden. Dank ihrer Therapieerfolge und 
ihres - im Vergleich zu anderen Therapieformen - zeitgemässen Ansatzes (zielgerichtete Therapie von relativ kurzer Dauer, klar umrissene Ziele, Evaluation der Therapieergebnisse) ist es der KVT in den letzten Jahren gelungen, sich sowohl bei Therapeuten als auch in der Öffentlichkeit erfolgreich durchzusetzen. Mediziner aller Fachrichtungen werden tagtäglich von unter Depressionen oder anderen psychischen Störungen leidenden Patienten konsultiert, die in einer Kognitiven Therapie erfolgreich behandelt werden könnten. Der vorliegende Beitrag zielte darauf $\mathrm{ab}$, den Betroffenen einen kurzen Überblick über die Möglichkeiten der KVT zu bieten und an einer Therapieausbildung interessierten Kollegen die Aktivitäten des Schweizerischen Vereins für Kognitive Psychotherapie (SVKoP) vorzustellen.

\section{Literatur}

- Clark D, Fairburn C (eds.). Science \& Practice of Cognitive Behaviour Therapy. Oxford: Oxford Press; 1997.

- André C. Les thérapies cognitives. Paris: Morisset; 2000.

- Cottraux J. Les thérapies cognitives. Paris: Retz; 2001.

- Mihaescu G, Séchaud MC, Delsignore A. Précis de thérapie comportementale et cognitive. Genève: Médecine et Hygiène; 1998.

- Guidano VF, Liotti G. Cognitive process and emotional disorders. New York: Guilford Press; 1983. 